Rev. Biol. Trop., 48(2/3): 587-590, 2000

www.ucr.ac.cr www.ots.ac.cr www.ots.duke.edu

\title{
Abortos de semillas de Enterolobium cyclocarpum (Mimosoideae): efecto de la posición relativa dentro del fruto
}

\author{
Federico Villalobos ${ }^{1}$ y Guillermo Bianchi ${ }^{2}$ \\ 1 Instituto Centroamericano de Biología y Conservación. Apartado 2398-2050 \\ San Pedro de Montes de Oca, San José, Costa Rica. \\ 2 Departamento de Biología, Facultad de Ciencias, Universidad de los Andes. Mérida. 5101. Venezuela.
}

Recibido 9-XII-1999. Corregido 9-II-2000. Aceptado 8-III-2000.

\begin{abstract}
We examined if seed abortion in Guanacaste tree fruits (Enterolobium cyclocarpum) is related to position within fruits, by establishing the abortion ratio in 150 fruits from 10 trees, collected in Santa Rosa National Park (Guanacaste, Costa Rica). Fruits were divided in basal, central and distal sections. We found marginal differences in abortion ratio between these sections (Anova, $p=0.058$ ), and also among trees $(p=0.01$ ). In general, the distal section had the greatest abortion ratio in relation to other sections. This abortion pattern could be caused by resource competition within siblings and not by genetic differences among them.
\end{abstract}

Key words: Enterolobium cyclocarpum, seed abortion, selective abortion, abortion patterns, position effects.

El aborto de flores, frutos y semillas es una estrategia común en numerosas especies vegetales (Bawa \& Webb 1984), en donde la fecundación de los óvulos no garantiza el desarrollo del embrión (Nakamura 1989). El aborto de semillas no se presenta de manera aleatoria ya que generalmente está asociada a la posición que éstas ocupan dentro del fruto (Bawa \& Webb 1984, Rocha \& Stephenson 1991). Este patrón se ha observado en numerosas leguminosas (Nakamura 1989, Bawa \& Buckley in Lee 1988).

En general, se considera que la competencia entre embriones es la causa de los aborto de semillas. La cercanía de los embriones a los recursos maternos le confieren ciertas ventajas competitivas (Lee 1988), al igual que la secuencia de fecundación de óvulos (Mogensen 1975) y el vigor del polen (Mulcahy \& Mulcahy 1983). Sin embargo, el aborto selectivo de embriones se presenta en un contexto de conflicto madre-hijo donde la planta madre tenderá a maximizar sus recursos en función de su adecuación (Haig \& Westoby 1988).
En Enterolobium cyclocarpum no se ha evaluado el efecto de la posición relativa del embrión en relación con la probabilidad de aborto, aunque Janzen (1982) sugirió que en esta especie las semillas abortadas presentaban una tendencia a encontrarse en el extremo distal del fruto y raramente sucede en los embriones ubicados entre semillas sanas. En esta investigación, evaluamos la tendencia de abortos de semillas en frutos de E. cyclocarpum.

Los frutos fueron recolectados en el Parque Nacional Santa Rosa (Guanacaste, Costa Rica) a mitad de 1998 (estación seca). Seleccionamos aleatoriamente diez árboles con "sombras de caída de fruto" bien definidas y recolectamos 15 frutos de cada individuo, tomando aquellos que se encontraban en un transecto de $1 \mathrm{~m}$ de ancho establecido aleatoriamente desde la base del árbol. Cada fruto fue dividido en tres regiones con igual número de óvulos: una región basal o peduncular, una central y una distal o estilar. Determinamos el número de óvulos, la posición de los abortos y el número 
de abortos por región enumerando desde el extremo peduncular hacia el extremo estilar

Con el objetivo de determinar el efecto de la posición de la semilla sobre su probabilidad de aborto realizamos un análisis de variancia anidado (con frutos dentro de árbol y posición dentro de fruto) para comparar la proporción de abortos por sección de fruto, excluyendo las otras fuentes de variación (árbol y fruto). $\mathrm{La}$ proporción de abortos por sección del fruto fue estimada dividiendo el número de abortos entre el número de óvulos. Esta variable fue transformada con la función arcoseno para obtener una distribución normal.

Existen diferencias en la proporción de abortos entre los árboles $(\mathrm{p}=0.01)$ y no se detectaron variaciones en la proporción de abortos entre los frutos de un árbol. Sin embargo, detectamos una diferencia marginal en el número de abortos entre las secciones del fruto $(p=0.058$, Cuadro 1$)$. La proporción de abortos fue mayor en la sección distal del fruto, mientras que la sección basal y central no difirieron significativamente (Fig. 1).

Nuestros observaciones referentes al número de semillas y al número de óvulos por fruto de E. cyclocarpum se encuentran dentro del ámbito descrito previamente para esta especie (Janzen 1982). Por otra parte, el patrón de aborto de semillas en esta especie coincide con el descrito por Wyatt (1981) en cinco especies de leguminosas tropicales, donde la pro- porción de abortos de embriones es mayor en el extremo distal del fruto.

Sin embargo, los patrones de aborto de semillas son variables en las leguminosas. En $\mathrm{Lu}$ pinus nanus y Medicago sativa, los abortos se concentran en los extremos del fruto (Horovitz et al. 1976). Bawa \& Webb (1984) estudiaron especies de árboles del bosque seco en Costa Rica, en las cuales la mayoría de éstos se concentraron en el extremo basal del fruto, patrón también presente en dos especies de Phaseolus (Nakamura 1989, Rocha \& Stephenson 1991).

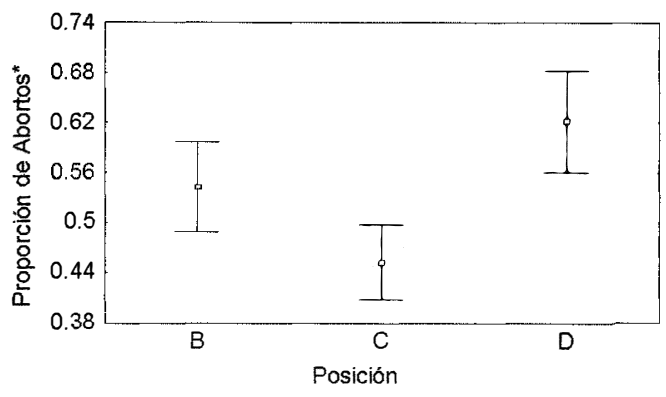

Fig.1. Promedio y dos errores estándar de la proporción de abortos según la posición basal (B), central (C) y estilar (E) en frutos de Enterolobium cyclocarpum. *Variable transformada, ver texto para más detalles.

Fig 1. Abortion ratio by seed position: basal (B), central (C) and stylar (E) on Enterolobium cyclocarpum fruits (mean and two standard errors) * Transformed variable, see text for further details.

\section{CUADRO 1}

Análisis de variancia anidado (GLM) para la posición y fruto en la proporción de abortos en E. cyclocarpum. $\left(r^{2}=0.463\right)$

Effect of seed position and fruit on abortion ration of Enterolobium cyclocarpum (Nested ANOVA, GML, $r^{2}=0.463$ )

$\begin{array}{llllll}\text { Efecto } & \text { SC } & \text { GL } & \text { CM } & \text { F } & \text { P } \\ \text { Árbol } & 2.130 & 9 & 2.237 & 2.377 & 0.0013 \\ \text { Frutos (Árbol) } & 16.663 & 140 & 0.119 & 1.195 & 0.108 \\ \text { Posición (Fruto) } & 4.405 & 30 & 0.147 & 1.474 & 0.050 \\ \text { Error } & 26.888 & 270 & 0.100 & & \end{array}$


Nakamaura (1989) determinó en Phaseolus vulgaris que el menor número de semillas en el extremo basal de las vainas era producto de la selección materna con base al genotipo del polen. Sin embargo, en el caso de E. cyclocakpum podría ser producto de la competencia entre embriones. Estudios genéticos preliminares en $E$. cyclocarpum utilizando marcadores enzimáticos (Rocha \& Lobo 1996, Hamrick \& Aldrich 1998) han revelado que los frutos son polinizados por un mismo tipo de polen. Estos resultados apoyan la observación que en esta especie, las poliadas tienen suficientes granos de polen para fecundar todos los óvulos de un fruto (Lobo com. pers.). Por lo tanto es posible que los embriones en la posición basal tengan un mayor acceso a los recursos maternos en la competencia con sus hermanos completos. Sin embargo, es necesario realizar estudios detallados sobre el proceso de polinización y fecundación así como de la paternidad de los embriones en una misma vaina.

\section{AGRADECIMIENTOS}

Agradecemos a F. Joyce y F. Chinchilla por su colaboración logística. A G. Campos por su colaboración en el análisis de resultados. A M. Quesada, N. Giannini y M. Blanco por sus comentarios al manuscrito.

\section{REFERENCIAS}

Bawa, K.S \& C.J. Webb. 1984. Flower, fruit and seed abortion in tropical forest trees: implications for the evolution of paternal and maternal reproductive patterns. Amer. J. Bot. 7: 736-751.
Haig, D. \& M. Westoby. 1988. Inclusive fitness, seed resources and maternal care. In: J. Lovett Doust, \& L. Lovett Doust (eds). 1988. Plant reproductive ecology: patterns and strategies. Oxford University, Oxford.

Hamrick, J.L. \& P.R. Aldrich. 1998. Temporal variation in the breeding structure of fragmented Enterolobium ciclocarpum populations. Amer. J. Bot. 85: 60.

Horowitz, A.; L. Meiri, \& A. Beiles. 1976. Effects of ovule position in fabaceous flowers on seed set and outcrossing rates. Bot. Gaz. 137: 250-254.

Janzen, D.H. 1982. Variation in average seed size and fruit seediness in a fruit crop of a Guanacaste tree (Leguminosae: Enterolobium cyclocarpum). Amer. J. Bot. 69: 1169-1178.

Mogensen, H.L. 1975. Ovule abortion in Quercus (Fagaceae). Amer. J. Bot. 62: 160-165.

Mulcahy, D.L. \& G.B. Mulcahy. 1983. Gametophytic selfincompatibility reexamined. Science 220: 1247-1251.

Nakamura, R. 1989. Seed abortion and seed size variation within fruits of Phaseolus vulgaris: pollen donor and resource limitation effests. Amer. J. Bot. 75: 1003-1010.

Lee, T.D. 1988. Patterns of fruit and seed production. In: Lovett Doust, J. \& L. Lovett Doust. 1988. Plant reproductive ecology: patterns and strategies. Oxford University, Oxford.

Rocha, O. \& J.A. Lobo. 1996. Genetic variation and differentiation among five populations of the Guanacaste tree (Enterolobium cyclocarpum Jacq) in Costa Rica. Int. J. Plant Sci. 157: 234-239.

Rocha, O. \& A.G. Stephenson. 1991. Effects of nonrandom seed abortion on progeny performace in Phaseolus coccineus L. Evolution 45: 1198-1208.

Wyatt, R. 1981. Components of reproductive output in five tropical legumes. Bull. Torrey Bot. Club. 108: 65-75. 